Zonen (Abb. 6, Bahn 2), die Phosphorylase lag in der b-Form vor, sie stammte von einem mit Schlagbolzen getöteten Tier. Ohne Adenosin-5'-monophosphat wurde Stärke nicht synthetisiert, selbst nicht bei so hoher Auftragsmenge, die mit AMP zu einer breiten verschmierten Zone führt (Abb. 6, Bahn 1).

Die Ratten waren mit Pentobarbital-Natrium eingeschläfert worden. Die Phosphorylase aus Herz war ohne Adenosin-5'-monophosphat inaktiv, aus Muskel sehr schwach aktiv, bei der Phosphorylase aus Leber ließ sich die Aktivität durch Zusatz von Adenosin-5'-monophosphat steigern (Abb. 7).
Die Wanderungsgeschwindigkeit der Leberphosphorylase entsprach der Wanderungsgeschwindigkeit der Phosphorylasen aus Herz und Muskel. Das Mol.-Gew. der Leberphosphorylase vom Hund entspricht in etwa dem Mol.-Gew. der b-Form der Muskelphosphorylase ${ }^{25}$ oder der Hälfte des Mol.Gew. der a-Form. Über mögliche elektrophoretische Umwandlungen von Phosphorylase a und Zwischenstufen wird zur Zeit gearbeitet.

Herrn P. HöPfEL danken wir für die sehr geschickte Mitarbeit bei der Durchführung der Versuche, der Deutschen Forschungsgemeinschaft (Bad Godesberg) für ihre finanzielle Unterstützung.

\title{
Homocylindrocarpidin und 17-Demethoxy-cylindrocarpidin, zwei neue Alkaloide aus Tabernaemontana amygdalifolia*
}

\author{
Hans Achenbach \\ Lehrstuhl für Biochemie der Pflanzen der Universität Freiburg i. Br. \\ (Z. Naturforschg. 22 b, 955-957 [1967] ; eingegangen am 10. Februar 1967)
}

\begin{abstract}
Chromatography of the extract of the root bark of Tabernaemontana amygdalifolia yielded two dihydroindole alkaloids. On the basis of spectral data, mainly conventional and high-resolution mass spectrometry, they were shown to be Homocylindrocarpidine (I) and 17-Demethoxy-cylindrocarpidine (II), respectively.
\end{abstract}

Durch wiederholte Chromatographie einer basischen Fraktion aus Tabernaemontana amygdalifolia wurden zwei weitere Alkaloide (I, II) isoliert. Die bei $120^{\circ}\left(10^{-3}\right.$ Torr $)$ destillierbaren Verbindungen haben folgende Daten (Tab. 1) :

\begin{tabular}{rccc}
\hline & $\begin{array}{c}\text { emp. } \\
\text { Formel } * *\end{array}$ & $\begin{array}{c}{[\alpha] \mathrm{D}_{\mathrm{D}}^{30}} \\
\left(\mathrm{CHCl}_{3}\right)\end{array}$ & $\begin{array}{c}\lambda_{\max }[\mathrm{nm}] \\
(\text { Äthanol 96\%) }\end{array}$ \\
\hline I & $\mathrm{C}_{24} \mathrm{H}_{32} \mathrm{~N}_{2} \mathrm{O}_{4}$ & $-82^{\circ}(c=1,7)$ & $219(\varepsilon=32800)$ \\
II & $\mathrm{C}_{22} \mathrm{H}_{28} \mathrm{~N}_{2} \mathrm{O}_{3}$ & $-49^{\circ}(c=1,9)$ & $256(\varepsilon=11400)$ \\
& & & $253(\varepsilon=19800)$ \\
& & & \\
\hline
\end{tabular}

Tab. 1. Physikalische Daten von Homocylindrocarpidin (I) und 17-Demethoxy-cylindrocarpidin (II).

Die abgeleiteten Strukturen des Homocylindrocarpidins (I) und 17-Demethoxy-cylindrocarpidins (II)

* 5. Mitteilung über „Massenspekrometrische Untersuchungen an Naturstoffen". - 4. Mitteilung: H. Аснenbach, Tetrahedron Letters [London] 1967, 1793.

** Die elementare Zusammensetzung des Molekülions und der wichtigsten Fragmente wurde mit einem doppelfokussierenden Massenspektrometer CEC 21-110 nach dem ,peak-matching'-Verfahren bestimmt (Direkteinlaß; $70 \mathrm{eV}$ ). basieren vorwiegend auf den Ergebnissen massenspektrometrischer Untersuchungen:

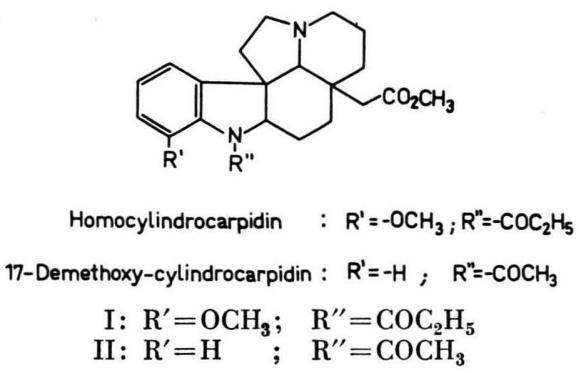

In den Massenspektren von I und II (Abb. 1) erkennt man ein Fragmentierungsverhalten, wie es nach den grundlegenden Arbeiten von Biemans und Mitarb. ${ }^{1-3}$ für Verbindungen mit dem Kohlenstoff-

Wir sind hierfür Herrn Dr. P. Schulze und der Fa. Bell \& Howell, Friedberg, zu Dank verpflichtet.

1 K. Biemann, M. Friedmann-Spiteller u. G. Spiteller, Tetrahedron Letters [London] 1961, 485.

2 K. Biemann, M. Friedmann-Spiteller u. G. Spiteller, J. Amer. chem. Soc. 85, 631 [1963].

3 K. Biemans, in: Mass Spectrometry, S. 315, McGraw-Hill, New York 1962. 


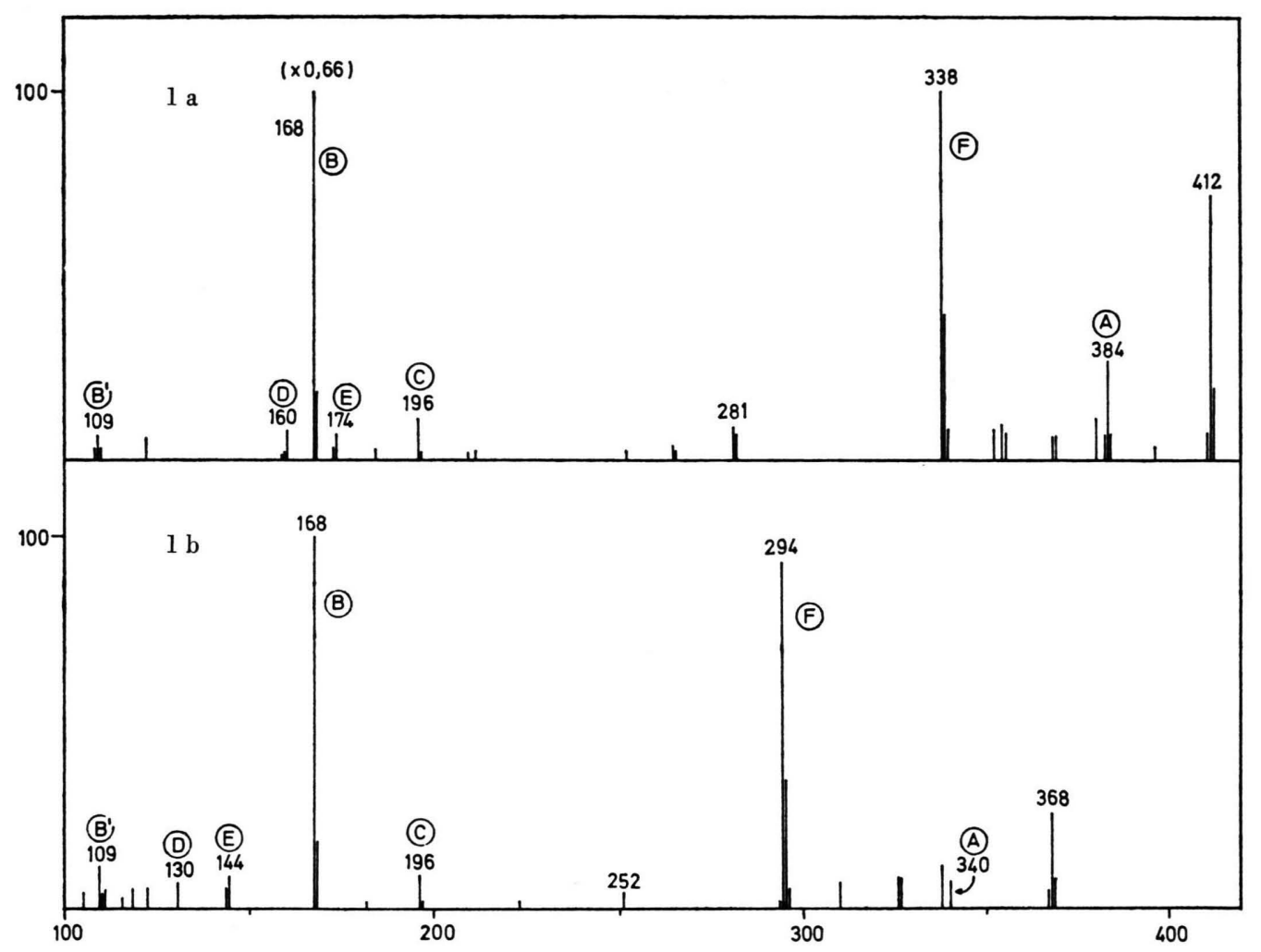

Abb. 1 a. Massenspektrum von I. 1 b. Massenspektrum von II.

gerüst des Aspidospermins ${ }^{4}$ charakteristisch ist (Abb. 2). Auch die durch hochauflösende Massenspektrometrie ermittelte elementare Zusammensetzung der wichtigsten Ionen steht hiermit im Einklang **.

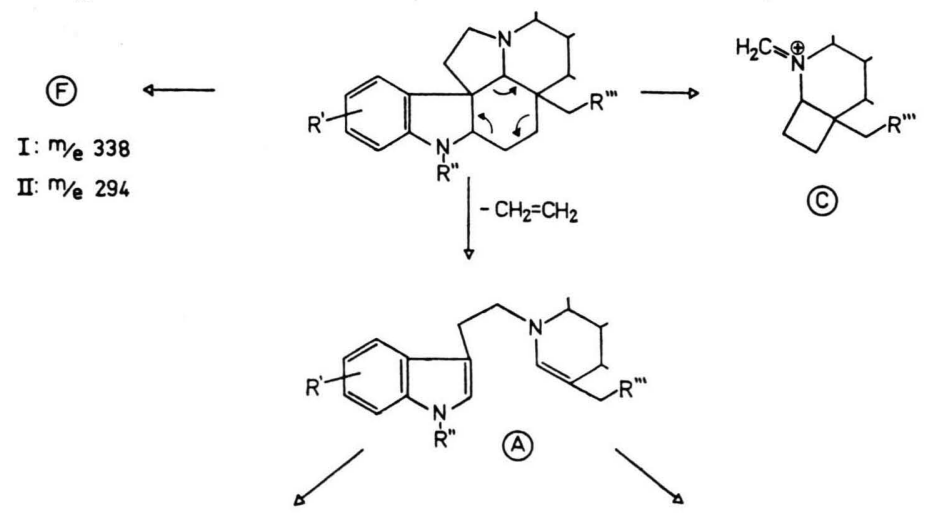

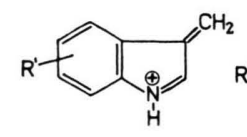

(D)<smiles></smiles>

(B)<smiles>CN1C=C([18O])CCC1</smiles>

Abb. 2. Massenspektrometrische Fragmentierung von Aspidospermin-Alkaloiden nach l. c. ${ }^{2}, 3$.

4 J. F. D. Mills u. S. C. Nyburg, Tetrahedron Letters [London] 11, 1 [1959]; H. Conroy, P. R. Broок u. Y. Aмirs, Tetrahedron Letters [London] 11, 4 [1959]. 
ionen entstehen. Als Erklärung hierfür bietet sich an die Abspaltung einer am C-5 des AspidosperminSkeletts fixierten Seitenkette $-\mathrm{CH}_{2}-\mathrm{CO}_{2} \mathrm{CH}_{3}$, die durch $\mathrm{McL}$ afferty-Umlagerung mit dem Wasserstoffatom am C-19 als $\mathrm{CH}_{2}=\mathrm{COH}-\mathrm{O}-\mathrm{CH}_{3}$ eliminiert wird.

Das dadurch entstehende Ion $(\mathrm{F})$ ist resonanzstabilisiert und in Übereinstimmung mit den Spektren nicht mehr zur Eliminierung von Aethen befähigt.

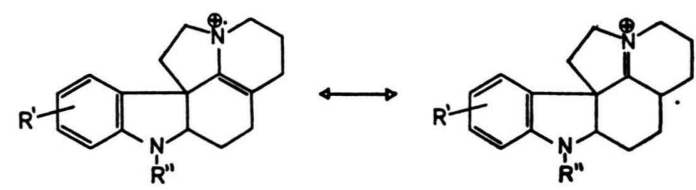

(ค)

Gestützt wird diese Überlegung durch die Fragmente (B), $\left(\mathrm{B}^{\prime}\right)$ und $(\mathrm{C})$, die für den alicyclischen Teil des Moleküls charakteristisch sind und im Massenspektrum sowohl von I als auch von II bei $m / e$ 168, 109 bzw. 196 auftreten.<smiles>COC(=O)CC1CCC[NH+](C)C1</smiles><smiles>CC1=CN(C)CCC1</smiles>

(B) $: m / e 168$
(B) : $m / \frac{109}{109}$

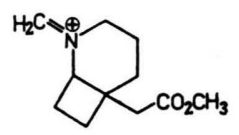

(C) : $m / e 196$
Demgegenüber erscheinen die für den Indolteil typischen Fragmente (D) und (E) im Massenspektrum von I jeweils $30 \mathrm{ME}$ höher als die entsprechenden Fragmente von II. Diese Verhältnisse zeigen, daß I eine aromatische Methoxylgruppe besitzt, während der Benzolring von II unsubstituiert ist.

I und II sind am Indolstickstoff acyliert $\left(v_{\mathrm{c}=0}\right.$ : $1660 \mathrm{~cm}^{-1}$ ). Durch Behandlung mit $\mathrm{HCl}$ gelingt die

5 A. J. Ewins, J. chem. Soc. [London] 105, 2738 [1914].

6 C. Djerassi, A. A. P. G. Archer, T. George, B. Gilbert u. L. D. Antonaccio, Tetrahedron [London] 16, 212 [1961].
Hydrolyse ${ }^{5}$. Remethylierung der gleichzeitig verseiften Estergruppe am C-21 mit Diazomethan führt zu den $\mathrm{N}_{\mathrm{a}}$-desacylierten Derivaten. Deren massenspektrometrisch bestimmte Molgewichte liegen 56 bzw. 42 ME niedriger als die Molgewichte von I bzw. II und fordern die Anwesenheit einer $\mathrm{N}_{\mathrm{a}}$-propionyl-Gruppe (in I) und einer $\mathrm{N}_{\mathrm{a}}$-acetyl-Gruppe (in II).

Unter den Bedingungen der $\mathrm{LiAlH}_{4}$-Reduktion verlieren I und II je 42 ME (Reduktion der Carbomethoxy-Gruppe: $-28 \mathrm{ME}$; Reduktion der $\mathrm{N}_{\mathrm{a}}$-acylGruppe: - 14 ME). In den Massenspektren der Reduktionsprodukte beobachtet man die erwartete Verschiebung der charakteristischen Fragmente.

Die Stellung der Methoxylgruppe in Position 17 des Benzolringes von I und die abgeleitete Struktur konnte bestätigt werden durch Vergleich von I mit dem Propionyl-Derivat des aus Cylindrocarpidin ${ }^{6}$ hergestellten Desacetylcylindrocarpidins. Beide Verbindungen waren identisch.

In Ubereinstimmung mit Formel I und II sind die KMR-Spektren: I: 3 arom. Protonen $(3,0-3,4 \tau)$; Singulett $\left(6,61 \tau\right.$ : $\left.\mathrm{CO}-\mathrm{O}-\mathrm{CH}_{3}\right)$; Singulett $(6,29 \tau$ : $\mathrm{O}-\mathrm{CH}_{3}$ (arom.)). II: 4 arom. Protonen (3,0 bis $3,4 \tau)$; Singulett $\left(6,62 \tau\right.$ : $\left.\mathrm{CO}-\mathrm{O}-\mathrm{CH}_{3}\right)$; Singulett $\left(7,90 \tau: \mathrm{CO}-\mathrm{CH}_{3}\right)$.

Neben I und II wurde auch Cylindrocarpidin aus Tab. amygdalifolia isoliert ${ }^{7}$.

Wir danken der Deutschen Forschungs gemeinschaft für eine Sachbeihilfe und der Stiftung Volkswagenwerk für die Bereitstellung eines Massenspektrometers vom Typ Atlas CH 4. Herrn Prof. K. Biemann, M.I.T., Cambridge (USA), und Herrn Dr. F. A. Hochstein, Chas. Pfizer (USA), danken wir für die Ubberlassung des Rohextraktes. Unser Dank gilt ferner Herrn Professor C. DJERAssi, Stanford University, Stanford, Cal. (USA), für eine authentische Probe Cylindrocarpiolin.

7 H. Achenbach, Tetrahedron Letters [London] 1967, 1793. 